\title{
Passive Immunization with Anti-Ganglioside Antibodies Directly Inhibits Axon Regeneration in an Animal Model
}

\author{
Helmar C. Lehmann, ${ }^{1,3 \star}$ Pablo H. H. Lopez, ${ }^{1 \star}$ Gang Zhang, ${ }^{1}$ Thien Ngyuen, ${ }^{1}$ Jiangyang Zhang, ${ }^{2}$ Bernd C. Kieseier, ${ }^{3}$ \\ Susumu Mori, ${ }^{2}$ and Kazim A. Sheikh ${ }^{1}$ \\ Departments of ${ }^{1}$ Neurology and ${ }^{2}$ Radiology, Johns Hopkins Medical Institutions, Baltimore, Maryland 21205, and ${ }^{3}$ Department of Neurology, Heinrich \\ Heine University, D-40225 Düsseldorf, Germany
}

\begin{abstract}
Recent studies have proposed that neurite outgrowth is influenced by specific nerve cell surface gangliosides, which are sialic acidcontaining glycosphingolipids highly enriched in the mammalian nervous system. For example, the endogenous lectin, myelinassociated glycoprotein (MAG), is reported to bind to axonal gangliosides (GD1a and GT1b) to inhibit neurite outgrowth. Clustering of gangliosides in the absence of inhibitors such as MAG is also shown to inhibit neurite outgrowth in culture. In some human autoimmune PNS and CNS disorders, autoantibodies against GDla or other gangliosides are implicated in pathophysiology. Because of neurobiological and clinical relevance, we asked whether anti-GD1a antibodies inhibit regeneration of injured axons in vivo. Passive transfer of anti-GD1a antibody severely inhibited axon regeneration after PNS injury in mice. In mutant mice with altered ganglioside or complement expression, inhibition by antibodies was mediated directly through GDla and was independent of complement-induced cytolytic injury. The impaired regenerative responses and ultrastructure of injured peripheral axons mimicked the abortive regeneration typically seen after CNS injury. These data demonstrate that inhibition of axon regeneration is induced directly by engaging cell surface gangliosides in vivo and imply that circulating autoimmune antibodies can inhibit axon regeneration through neuronal gangliosides independent of endogenous regeneration inhibitors such as MAG.
\end{abstract}

Key words: gangliosides; axon regeneration; anti-ganglioside antibodies; Guillain-Barré syndrome; passive transfer; animal model

\section{Introduction}

Axon regeneration is a response of injured nerve cells that is critical for the restoration of structure and function after PNS or CNS injuries; this response is key to recovery from numerous neurological disorders. Defining the molecules and signal transduction pathways that prevent regeneration of injured axons can provide key insights that may allow development of therapeutic approaches to enhance axon growth in neurological diseases.

Gangliosides are major cell surface determinants and the predominant sialoglycoconjugates of which GM1, GD1a, GD1b, and GT1b are the most abundant gangliosides in the adult mammalian nervous system (Yu and Saito, 1989). These complex gangliosides are structurally related and contain a ceramide lipid anchor, a neutral tetrasaccharide core (Gal $\beta 3$ GalNAc $\beta 4$ Gal $\beta 4$ Glc), and one or more sialic acids. They are biosynthesized by the sequential action of a series of specific glycosyltransferases (see Fig. 1) (van Echten and Sandhoff, 1993). Neurobiological roles of the major nervous system gangliosides are not completely de-

\footnotetext{
Received Sept. 14, 2006; revised Nov. 13, 2006; accepted Nov. 13, 2006.

This work was supported by grants from the National Institutes of Health (NS42888), the Guillain-Barré Syndrome Foundation, and the Muscular Dystrophy Association. We thank Dr. Pamela Talalay for editorial discussion, Drs. John Griffin and Ronald Schnaar for helpful suggestions and advice, and Dr. Richard Proia (National Institutes of Health, Bethesda, MD) for providing breeding pairs of Siat8a-null mice.

${ }^{*}$ H.C.L. and P.H.H.L. contributed equally to this work.

Correspondence should be addressed to Dr. Kazim A. Sheikh, Department of Neurology, Johns Hopkins Hospital, 600 North Wolfe Street, 509 Pathology Building, Baltimore, MD 21205. E-mail: ksheik@jhmi.edu.

DOI:10.1523/JNEUROSCI.4017-06.2007

Copyright $\odot 2007$ Society for Neuroscience $\quad$ 0270-6474/07/270027-08\$15.00/0
}

fined. Previous work indicates that complex gangliosides contribute to long-term axon and myelin stability (Sheikh et al., 1999; Pan et al., 2005). Recent in vitro studies propose that neurite outgrowth can be inhibited by engaging major nervous system gangliosides GD1a and/or GT1b in the context of myelinassociated glycoprotein (MAG) (Vinson et al., 2001; Vyas et al., 2002; Fujitani et al., 2005). Clustering cell surface gangliosides GD1a or GT1b with multimeric ligands (precomplexed IgG or IgM antibodies), in the absence of MAG, also induces inhibition of neurite outgrowth (Vyas et al., 2002; Fujitani et al., 2005). Whether inhibition of axon regeneration can be induced through cell surface gangliosides (without the presence of MAG) in an animal model remains to be examined.

Complex gangliosides have also been implicated as target antigens in autoimmune disorders of the PNS and CNS including multiple sclerosis (Sadatipour et al., 1998; Willison and Yuki, 2002). For example, antibodies (Abs) against GD1a ganglioside have strongest association with some forms of Guillain-Barré syndrome (GBS) (Willison and Yuki, 2002; Hughes and Cornblath, 2005), which is the commonest cause of acute flaccid paralysis worldwide. GBS comprises a group of clinically and pathophysiologically related, acute monophasic neuropathic disorders of autoimmune origin that can be demyelinating or axonal (Willison and Yuki, 2002; Hughes and Cornblath, 2005). Clinical studies indicate that anti-GD1a Abs in some adult patient with GBS are associated with poor prognosis and/or incomplete recovery (Yuki et al., 1993; Carpo et al., 1999; Press et al., 2001). 
Patients with incomplete recovery have some degree of failure of axon regeneration and target reinnervation (Brown and Feasby, 1984). Because the mammalian PNS usually regenerates after injury, the mechanisms underlying failure of axon regeneration in such cases with permanent neurologic sequelae are unclear.

Because of its neurobiological and clinical relevance, we asked whether engaging GDla ganglioside with Abs can inhibit regeneration of injured axons. We examined this issue in an animal model of peripheral nerve injury, in which rapid clearance of myelin and associated myelin inhibitors like MAG, typically allows effective peripheral nerve regeneration (Schafer et al., 1996; Filbin, 2003).

\section{Materials and Methods}

Anti-ganglioside monoclonal antibody. A previously well characterized monoclonal antibody (mAb) GD1a/GT1b IgG2b (GD1a/GT1b-2b) was used for all passive transfer studies because it binds to both myelinated and unmyelinated axons in peripheral nerves (Gong et al., 2002), its pathobiological effects depend on expression of corresponding specific gangliosides (Sheikh et al., 2004; Zhang et al., 2004; Goodfellow et al., 2005), and it induces mild complementdependent neuropathy in an animal model (Sheikh et al., 2004). The generation, specificity, production, and purification of this mAb were reported previously (Lunn et al., 2000; Gong et al., 2002). GD1a/GT1b-2b in ascites (Covance, Princeton, NJ) $(n=4)$, or hollow fiber supernatant (Johns Hopkins Cell Culture Facility) $(n=3)$, or purified $\mathrm{Ab}(n=2)$, was used for the passive immunization of wildtype animals. Ascites containing GD1a/GT1b-2b was used for all studies in transgenic or mutant mice. An irrelevant mouse IgG-2b mAb HB-94 (Zhang et al., 2004) or mouse IgGs (Alpha Diagnostic, San Antonio, TX) were used as sham or control Abs.

Mice. All sciatic nerve crush studies were done on 12- to 16-week-old wild-type (C57BL/6) and mutant mice (Galgt1-null, Siat8a-null, or C5deficient). Galgt1-null mice (UDP- $N$-acetyl-D-galactosamine:GM3/ GM2/GD2 synthase; EC 2.4.1.92) mice lack complex gangliosides, including GD1a, and express only simple gangliosides GM3 and GD3 (Liu et al., 1999) (Fig. 1). We also examined a complementary transgenic mouse lacking the key enzyme Siat8a (CMP-sialic acid:GM3 a-2,8sialyltransferase or GD3 synthase; EC 2.4.99.8) required for synthesis of b-series gangliosides. Siat8a-null mice express a-series GM3, GM2, GM1, and GD1a, but not b-series GD3, GD2, GD1b, and GT1b (Kawai et al., 2001) (Fig. 1). Notably, ganglioside GD1a is overexpressed in the nervous system of these animals (Kawai et al., 2001; Goodfellow et al., 2005). C5-deficient (DBA/2J; The Jackson Laboratory, Bar Harbor, ME) animals have undetectable levels of C5, low levels of C3, and cannot form terminal membrane attack complex (C5b-9) (Cinader et al., 1964). Use of these mutants allowed examining the role of ganglioside expression and terminal membrane attack complex in this model of inhibited axon regeneration. All experimental procedures were approved by the Johns Hopkins University Animal Care and Use Committee.

Sciatic nerve crush model. A standardized mouse sciatic nerve crush model was used. Left sciatic nerves were crushed $35 \mathrm{~mm}$ rostral to the middle toe for $30 \mathrm{~s}$ with fine forceps on day 0 . Separation of proximal and distal endoneurial contents indicated complete crush. Animals were administered six doses of GD 1a/GT1b-2b [1 ml of ascites $(n=4)$ or hollow fiber supernatant $(n=3)$ containing $\sim 3 \mathrm{mg}$ of $\mathrm{Ab}$, or $\sim 4 \mathrm{mg}$ of purified $(n=2) \mathrm{Ab}$ ], or control Abs $(n=7)$ (3-4 mg of purified mouse IgGs) on days $3,5,7,9,11$, and 13 after surgery by intraperitoneal route. Approx- imately $18-24 \mathrm{mg}$ of $\mathrm{mAb}$ per animal was administered over this $12 \mathrm{~d}$ period. Because morphological changes in animals receiving ascites, hollow fiber supernatant, or purified Abs were similar, these animals were analyzed as a single group. On days 15-16 after nerve crush, a magnetic resonance imaging (MRI) of the calf muscles and electrophysiology were obtained on selected animals; tissues and sera were harvested for morphological and serological analysis from all animals between days 16 and 18. Serological studies for GM1, GD1b, GD1a, and GT1b ganglioside binding were done by ELISA as described previously (Lunn et al., 2000).

Morphometry. Mice were perfused with a mixture of $3 \%$ glutaraldehyde and 4\% paraformaldehyde. Sciatic and tibial nerves and lumbar spinal cords were harvested and immersion-fixed overnight before processing. For morphology, four segments of the crushed sciatic nerves were analyzed: $\mathrm{S} 1,10 \mathrm{~mm}$ above the crush site; $\mathrm{S} 2$, immediately distal to crush site; $\mathrm{S} 3,10 \mathrm{~mm}$ distal to the crush site (S1-S3 sciatic nerve segments); and S4, $20 \mathrm{~mm}$ distal to the crush site (tibial nerve segment) (see Fig. $2 \mathrm{~A}$ ). These segments were embedded in Epon, and $1 \mu \mathrm{m}$ cross sections were stained with toluidine blue as described previously (Sheikh et al., 1999, 2004). Sections from nerve segments S3, S4, and S1 were used for quantification at light level $(60 \times$ lens) by a motorized stage and stereotactic imaging software (Stereo Investigator, version 5). We counted all myelinated regenerating sprouts (S3-S4) or uninjured axons ( $\mathrm{S} 1$, above the crush site) in a single whole cross section of the nerve. Nerve sections immediately distal to the crush site (S2) were examined by both light microscopy and electron microscopy (EM). Epon-embedded cross sections were used for quantifying mean caliber of regenerating axons/sprouts in S2 segments. Caliber was calculated from the diameter of a circle with an area equivalent to that of each axon/sprout, as described previously (Sheikh et al., 1999). Because regenerating axon tips are not myelinated, dystrophic sprouts were arbitrarily defined as sprouts/axons $>1 \mu \mathrm{m}$ in diameter that were without myelin. Unmyelinated fibers (UMFs) were quantified by EM, as described previously (Murinson et al., 2005). Briefly, systematic random sampling (total area sampled, $1.5-2 \%$ of total nerve area) was done on ultrathin sections of sciatic (S3) and tibial (S4) segments, selected areas were photographed and digitized, and UMFs were counted at $15,000 \times$ magnification. 
Paraffin-embedded sections of L4-L5 spinal cord segments were examined for morphological changes in motor neurons.

Sciatic nerve ligature model. We used a sciatic nerve ligature model to examine $\mathrm{Ab}$ binding to injured axons because it allows direct comparison of injured tips in a defined region of the nerve in both GD1a/GT1b-2band sham-treated groups, in contrast to the crush model, in which dystrophic axon tips or normally regenerated axons are confined to S2 segments in GD1a/GT1b-2b-treated and sham Ab-treated groups, respectively. This precluded direct comparison of Ab binding to injured tips between the two groups. Furthermore, regeneration of axons in nerve segments distal to the crush site is known to restore the blood-nerve barrier (BNB) (Seitz et al., 1989), again precluding direct comparison between two groups because of differences in BNB permeability. Sciatic nerves of C57BL/6 (wild-type) mice were tightly ligated with 6.0 silk sutures at midthigh level and transected below the sutures. Animals were divided into two groups. One group received GD1a/GT1b-2b and the other group received a sham $\mathrm{Ab}$ as described for sciatic nerve crush model. Sciatic nerves (including the ligated tips) were harvested on day 16 after the surgeries and fixed with $4 \%$ paraformaldehyde for $24 \mathrm{~h}$. These nerves were cryoprotected with sucrose, longitudinally sectioned, immunostained with anti-mouse IgG/IgG2b or both anti-mouse IgG/IgG2b and anti- $\beta$-III tubulin Abs, and analyzed by fluorescent or confocal microscopy.

Electrophysiology. Compound muscle action potential (CMAP) amplitudes were recorded with a PowerLab signal acquisition set-up (ADInstruments, Grand Junction, CO) in the tibial innervated muscles (sole/ flexor compartment) of the hindpaws at baseline (before nerve crush) and before killing animals. Mice were anesthetized with $1.5 \%$ isoflurane and placed on heating pad at $37^{\circ} \mathrm{C}$, and their body temperature was monitored with a rectal probe and maintained at $35-37^{\circ} \mathrm{C}$. Recording needle electrodes were inserted in the sole of the foot and the sciatic nerve was stimulated with needle electrodes at the sciatic notch.

MRI. Live anesthetized animals were imaged by an 11.7 Tesla nuclear magnetic resonance spectrometer. Three-dimensional (3D) magnetic resonance images of calf region (between knee and ankle) were acquired with resolution of $0.1 \times 0.1 \times 0.4 \mathrm{~mm}$ with no gap between slices. Images were segmented manually or semiautomatically with software Amira (Mercury Computer Systems, Chelmsford, MA). The entire volume of the lower limb was segmented first by using a simple region growing technique, which includes both bone and muscle tissue. Volumes of bone and any fat were then manually segmented and subtracted from the total volume to give the total muscle volume. From 3D images of the lower limb, volumetric measurements of all muscle groups were obtained by multiplying the number of pixels that belong to a muscle/muscle group with the size of each individual pixel.

Statistical analyses. Data are reported as mean \pm SEM. Differences between groups were examined for statistical significance by Wilcoxon's rank sum (Mann-Whitney) test, because data had non-normative distribution. A value of $p<0.05$ was considered significant.

\section{Results \\ Inhibition of axon regeneration by passive transfer of anti-ganglioside Abs}

We first examined whether anti-GDla Abs inhibit axon regeneration in sciatic nerve crush model. ELISA confirmed that antiganglioside $\mathrm{mAb}$ reached the circulation because sera obtained from mice administered GD1a/GT1b-2b reacted only with GD1a (titers, 1:6400-12,800) and GT1b gangliosides (titers, 1:32006400). Morphological studies showed that the number of regenerating axons in sciatic and tibial nerves (S3 and S4 segments, respectively) decreased dramatically compared with those in controls (sham Ab-treated regenerating nerves) (Fig. $2 B-E$ ). Morphometric analysis showed $>10$ - and 30 -fold decreases in numbers of regenerating myelinated fibers (MFs) in GD1a/GT1b-2btreated mice compared with controls in sciatic (S3) and tibial (S4) segments, respectively (Fig. $2 F$ ). EM morphometry showed that the number of regenerating UMFs in GD1a/GT1b-2b-treated
A

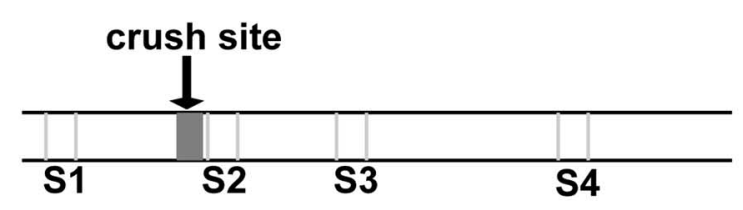

\section{Sciatic nerve (SN) Tibial nerve (TN)}
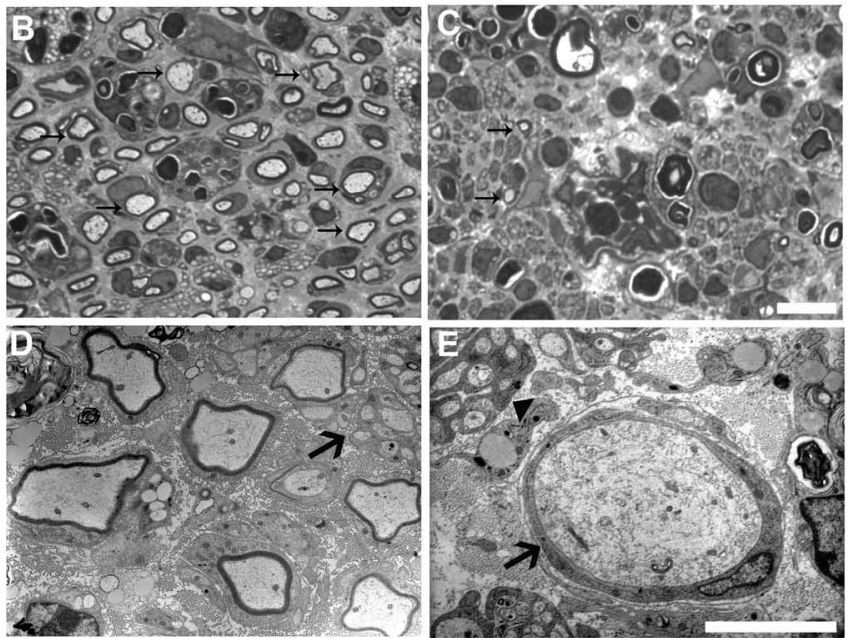

$\mathbf{F}$

G
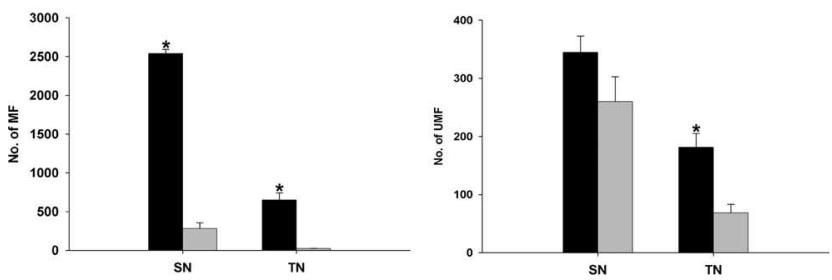

Figure 2. Anti-glycan Ab-mediated inhibition of axon regeneration in peripheral nerves. $\boldsymbol{A}$, Schematic diagram showing the site of nerve crush and its relationship with different nerve segments (S1-S4) as outlined in the text. $\boldsymbol{B}-\boldsymbol{E}$, Light (scale bar, $20 \mu \mathrm{m})(\boldsymbol{B}, \boldsymbol{C})$ and electron (scale bar, $2 \mu \mathrm{m})(\boldsymbol{D}, \boldsymbol{E})$ micrographs of sciatic nerve $S 3$ segments. $\boldsymbol{B}$, Many regenerating myelinated axons in sham-treated animal (arrows). C, Rare regenerating nerve fibers in GD1a/GT1b-2btreated animal (arrows). D, Both myelinated and unmyelinated fibers (arrow) are present in sham-treated animals. $\boldsymbol{E}, \mathrm{GD1}$ a/GT1b-2b-treated nerve with a dystrophic sprout (arrow) and unmyelinated fibers (arrowhead), but no myelinated fibers in this field. $\boldsymbol{F}$, Significant decrease in numbers of MFs in GD1a/GT1b-2b-treated (gray bars; $n=9$ each) sciatic (SN) (283 \pm 74 ) and tibial (TN) $(21 \pm 3)$ nerves compared with sham Ab-treated (black bars; $n=7$ each) sciatic (2540 \pm 52$)$ and tibial (651 \pm 92$)$ nerves. G, Significant decrease in numbers of UMFs in GD1a/GT1b-treated nerves at the tibial but not the sciatic level ( $n=5$ each of sciatic and tibial nerves/group). ${ }^{*} p<0.05$. Error bars indicate SEM.

nerves was nonsignificantly reduced in the sciatic segment (S3), whereas UMFs in the tibial segment (S4) were significantly decreased compared with those in controls (Fig. 2G). In GDla/ GT1b-2b-treated nerves, there was no active degeneration of nerve fibers above the crush site, and the total numbers of nerve fibers in these (S1) segments $(3773 \pm 152 ; n=3)$ and those of control nerves ( $3994 \pm 146 ; n=3$ ) were not significantly different. Motor neurons in spinal cord sections showed reactive changes on the injured (nerve crush) side in both GD1a/GT1b-2b and control mice without neuronal loss (data not shown), also reported previously (Sheikh et al., 2004). Therefore, GD1a/ GT1b-2b Ab inhibited regeneration of both myelinated and unmyelinated nerve fibers in the PNS without affecting the survival 

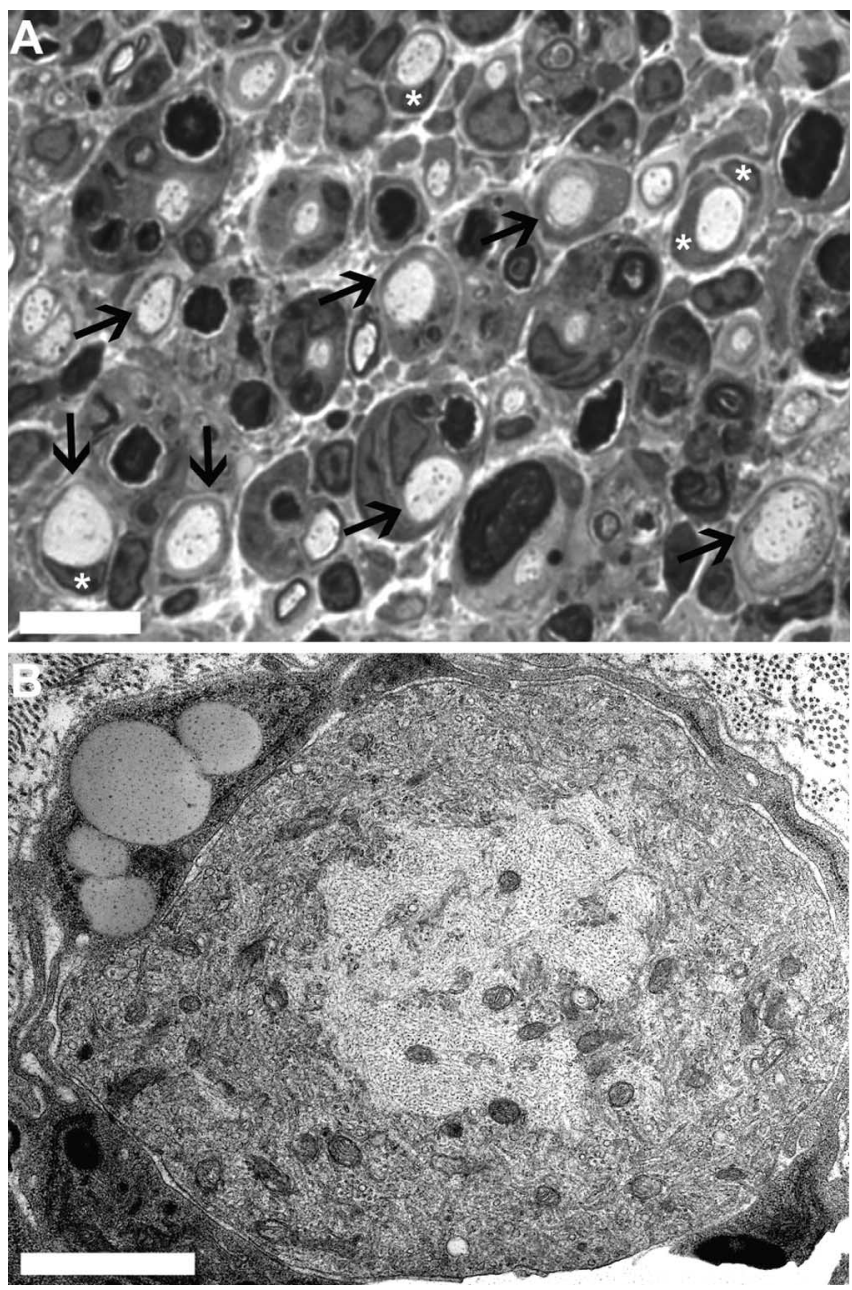

Figure 3. Anti-glycan Abs induce formation of dystrophic regenerating sprouts. $\boldsymbol{A}$, Light micrograph showing abortive sprouting characterized by dystrophic sprouts (arrows); these sprouts are not myelinated and are surrounded by Schwann cell nuclei $\left({ }^{*}\right)$ (scale bar, $20 \mu \mathrm{m}$ ). $\boldsymbol{B}$, EM showing dystrophic growth cone with a core of neurofilaments and a surrounding pellet with membranous organelles (scale bar, $2 \mu \mathrm{m}$ ).

of nerve fibers in the proximal stump or parent neurons in the spinal cord. Notably, myelinated axons were more susceptible to Ab-mediated inhibition than were unmyelinated axons, but the cellular/molecular basis of this differential susceptibility is not clear.

\section{Anti-ganglioside Abs induce abortive dystrophic sprouting}

We examined GD1a/GT1b-2b-treated nerves to determine the morphology of inhibited axon tips because stalled growth cones/ axon tips are known to undergo dystrophy or collapse (Silver and Miller, 2004). Histological studies showed that S2 segments (adjacent to the crush site) of GD1a/GT1b-2b-treated nerves were full of dystrophic sprouts that were almost always surrounded by Schwann cells (Fig. 3A). EM indicated that these sprouts were surrounded by Schwann cell processes and they were filled with whorls of neurofilaments, a number of which ended in massive axon terminals containing membranous organelles (Fig. 3B). Morphometry showed significant $(p<0.05)$ increase in percentage of dystrophic sprouts in S2 nerve segments of GD1a/GT1b2b-treated animals ( $57 \pm 6 ; n=3$ ) were dystrophic compared with controls $(5 \pm 1 ; n=3)$. These findings suggest that there is severe inhibition of axon regeneration rather than nonspecific
Ab-mediated destructive injury of nerve fibers, because most fibers showed morphology reminiscent of stalled growth cones called dystrophic bulbs, originally described by Ramon y Cajal (1928). The dystrophic growth cones, similar to dystrophic endings formed in vivo, can also be induced experimentally with other inhibitors of axon regeneration such as proteoglycans (Tom et al., 2004).

\section{Anti-ganglioside Abs bind to injured axon tips}

We next examined whether GD1a/GT1b-2b Ab binds to injured axon tips in sciatic nerve ligature model (see Materials and Methods). Our immunocytochemistry studies showed that the sciatic nerve region immediately above the ligatures had significantly higher deposition of IgG in GD1a/GT1b-2b Ab-treated groups compared with controls. Most of the increased staining was in the tip region where injured/transected axons are confined (gradient of staining along the nerve) (Fig. $4 A-C$ ). Double labeling studies showed that most axons had IgG deposition in the GD1a/GT1b2b-treated group compared with controls (Fig. 5). These studies show that GD1a/GT1b-2b Ab binds to injured axon tips, which have greater access to the circulating Abs because of breakdown of the BNB. These immunocytochemical studies in conjunction with quantitative data on S1 segments suggest that the inhibitory effects are mediated at the level of injured axon tips and are not attributable to more proximal $\mathrm{Ab}$ binding.

\section{Inhibition of target reinnervation by passive transfer of anti-ganglioside Abs}

Innervation of target muscles by regenerating axons was evaluated by measuring CMAP amplitudes in the hindpaw and volumetric MRI of calf muscles. Sciatic nerve conductions showed that CMAP amplitudes were delayed and dispersed (consistent with regeneration) in controls, but not in GD1a/GT1b-2btreated animals, at day 16 after the nerve crush (Fig. 6A). MRI volumetric measurements (Fig. $6 B$ ) of total calf musculature on day 16 after the sciatic nerve crush showed a significant $(p<$ $0.05)$ decrease in total muscle volume in GD1a/GT1b-2b-treated animals $\left(152 \pm 8 \mathrm{~mm}^{3} ; n=3\right)$ compared with those in control group $\left(183 \pm 6 \mathrm{~mm}^{3} ; n=3\right)$. Overall, these studies show that target (muscle) reinnervation was decreased in GD1a/GT1b-2btreated animals, as reflected by absent CMAP amplitudes and hindlimb atrophy.

\section{Anti-ganglioside $\mathrm{Ab}$ induced inhibition of axon regeneration} in transgenic mice with altered ganglioside expression Two different strains of transgenic mice were examined to determine whether GD1a/GT1b-2b-mediated inhibition of axon regeneration depends on expression of appropriate gangliosides in the nerves and is not attributable to cross-reaction with glycoprotein(s). The number of MFs in Galgt1-null animals (lacking complex gangliosides including GD1a) (Fig. 1) was not affected by GD1a/GT1b-2b administration (Fig. 7A), indicating that expression of complex gangliosides is necessary for the GDla/ GT1b-2b-mediated inhibition of axon regeneration. There were fewer regenerative MFs in tibial segments of the Galgt1-null mice compared with wild-type animals (Fig. $2 F$ ). It is possible that lack of GM1 ganglioside, extensively purported to be neurotrophic (Mutoh et al., 1995), in Galgt1-null animals is responsible for delayed regeneration in Galgt1-null mice.

Systemic administration of GD1a/GT1b-2b mAb in Siat8anull animals (overexpressing GDla and lacking b-series gangliosides) (Fig. 1) caused severe inhibition of axon regeneration compared with that in controls at both sciatic (S3) and tibial (S4) 

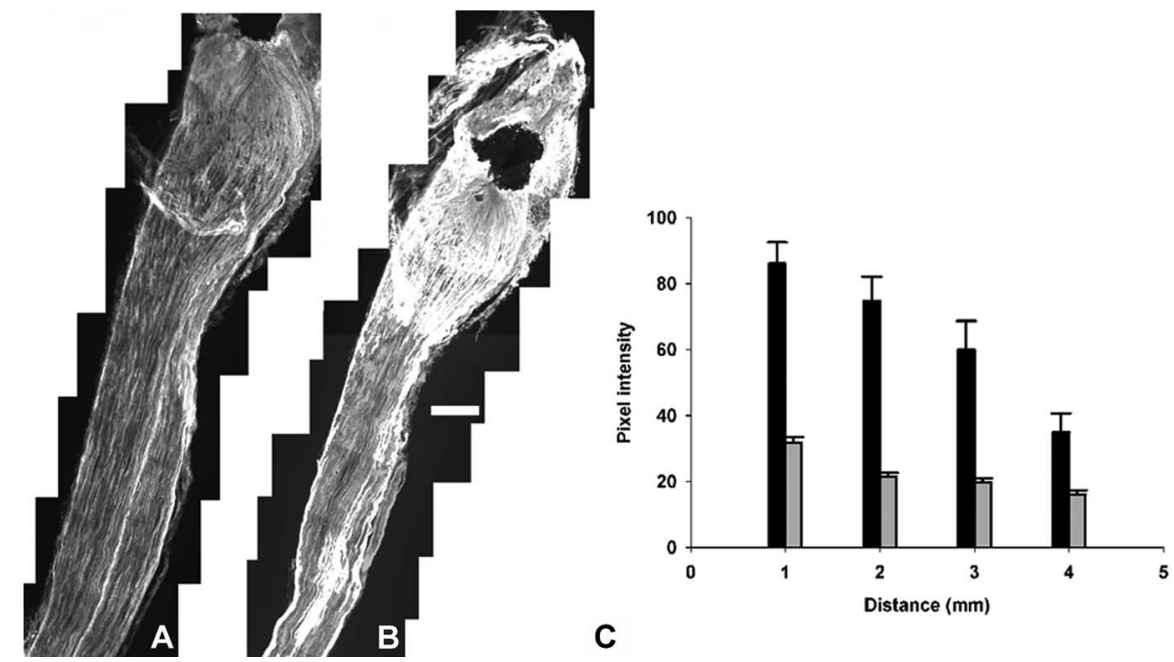

Figure 4. IgG deposition in nerve ligation model. $\boldsymbol{A}, \boldsymbol{B}, \mathrm{Increased} \lg \mathrm{G}$ deposition in GD1a/GT1b-2b-treated nerves ( $\boldsymbol{B})$ compared with sham Ab-treated nerves $(\boldsymbol{A})$. Scale bar: (in $\boldsymbol{B}) \boldsymbol{A}, \boldsymbol{B}, 0.5 \mathrm{~mm}$. C, Quantification shows a gradient of $\mathrm{g} G$ staining from tip $(1 \mathrm{~mm})$ to more proximal segments of the nerve $(4 \mathrm{~mm})$ in GD1a/GT1b-2b-treated group (black bars) compared with sham-Ab-treated group (gray bars). Error bars indicate SEM.
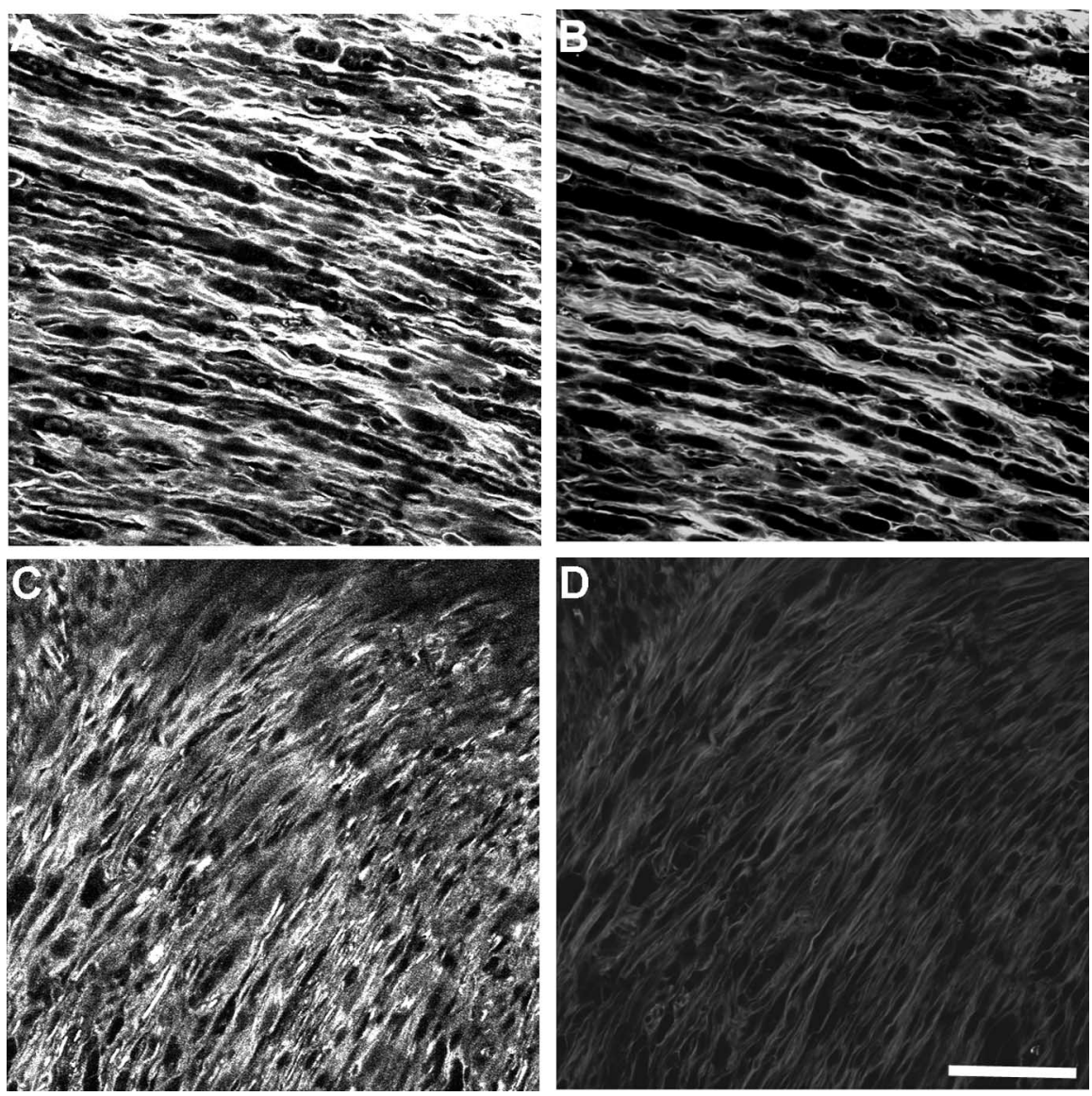

Figure 5. GD1a/GT1b-2b binds to injured axon tips. GD1a/GT1b-2b-treated $(\boldsymbol{A}, \boldsymbol{B})$ and sham-treated $(\boldsymbol{C}, \boldsymbol{D})$ nerves. Double labeling studies for $\beta$-III tubulin $(A, C)$ and immunoglobulins $(B, D)$ show $\lg G$ binding to axons in GD1a/GT1b-2b-treated nerves compared with controls. Scale bar, $20 \mu \mathrm{m}$.

levels (Fig. 7B). GD1a/GT1b-2b-mediated inhibition of axon regeneration thus requires expression of complex gangliosides and this $\mathrm{Ab}$ inhibits axon regeneration through its interaction with GDla ganglioside.
Anti-ganglioside Ab induced inhibition of axon regeneration in mutant mice deficient in C5 component of complement

GD1a/GT1b-2b mAb used in these studies is an IgG2b that can fix complement, raising the possibility that complementmediated cytolytic injury may be involved in inhibition of nerve regeneration. We examined this issue in a mutant mouse strain deficient in the C5 component of complement. The numbers of regenerating MFs in GD1a/GT1b-2b-treated animals were decreased $>2$-fold at sciatic (S3) and $\sim 10$ fold at the tibial level (S4) compared with those in control animals (Fig. 7C).

GD1a/GT1b-2b-mediated inhibition in C5-deficient animals was relatively less than that in C5-sufficient mice (C57BL/6) (Fig. 2). Notably, significantly more regenerating axons were found in sham $\mathrm{Ab}$ treated C5-deficient animals than in their sham Ab-treated C5-sufficient counterparts. It is unclear whether more robust inhibition in C5-sufficient mice can be attributed to Ab-mediated complementdependent injury, or conversely, less inhibition in C5-deficient animals indicates higher regenerative response in this strain as a result of C5 deficiency and/or other factors related to different backgrounds.

Nevertheless, it is clear that GD1a/ GT1b-2b Ab led to highly significant inhibition of axon regeneration in mutant mice deficient in complement.

\section{Discussion}

Our results demonstrate that engaging the axonal surface ganglioside GD1a, by using an anti-ganglioside $\mathrm{mAb}$, directly inhibits regeneration of peripheral nerve axons. This inhibition was induced in an animal model of peripheral nerve injury, in which rapid clearance of myelin debris and myelin-associated molecules such as MAG allows effective peripheral nerve regeneration (Schafer et al., 1996; Filbin, 2003). These studies imply that diseaseassociated autoimmune Abs directed against axonal targets of regeneration inhibitors can limit the recovery of injured axons. Furthermore, acquired autoimmune Abs to axonal targets can mediate inhibition of regeneration independent of endogenous axon regeneration inhibitors. Because inhibition of axon regeneration was demonstrated in an animal model by systemic administration of anti-GDla Abs, it may be inferred that circulating Abs may be pathogenic to axon regeneration in autoimmune diseases.

The conclusion that GDla ganglioside can mediate antiganglioside $\mathrm{Ab}$-induced inhibition of axon regeneration is sup- 
ported by the following observations: (1) GD1a/GT1b-2b mAb, used in the current study, requires expression of gangliosides for its pathobiological effects on nerve cells in animal and in vitro models (Sheikh et al., 2004; Zhang et al., 2004; Goodfellow et al., 2005); (2) Galgt1-null mice lacking the terminal "NeuAc $\alpha 3$ Gal $\beta 3$ GalNAc" motif on complex gangliosides including GD1a, but not on glycoproteins, are not susceptible to GD1a/GT1b-2b-mediated inhibition; (3) Siat8a-null mice, which do not synthesize GT1b ganglioside but abundantly express GD1a, show inhibition of axon regeneration comparable with that in wild-type animals; and (4) previous studies indicate that relative to other major gangliosides the synthesis of GD la ganglioside increases and it becomes the major newly synthesized ganglioside on regenerating sciatic nerves (GuzmanHarty et al., 1990). The presence of stalled growth cones/dystrophic sprouts indicates that anti-GDla Abs inhibit axon regeneration in the denervated segment of sciatic nerve up to $18 \mathrm{~d}$ after injury (latest time point examined). Longitudinal studies will be required to address the question whether clearance of Abs from circulation allows regeneration of inhibited nerve fibers in this model. Overall, this concept of inhibition of axon regeneration through gangliosides is also supported by previous work showing that Abs against GM1 ganglioside can inhibit regeneration in nonmammalian neurons (Schwartz and Spirman, 1982; Sparrow et al., 1984).

Our findings suggest that inhibition of axon regeneration through GDla can be induced by ganglioside ligands such as anti-ganglioside Abs independent of MAG. In comparison, MAG-mediated inhibition may be more complicated. In addition to gangliosides, Nogo receptor (NgR) has been shown to mediate MAGmediated inhibition (Domeniconi et al., 2002; Liu et al., 2002; Wang et al., 2002). Previous studies with MAG and gangliosides do not directly address the possibility that experimental alterations of ganglioside expression in nerve cells may modulate the interactions of MAG with $\mathrm{NgR}$ to induce inhibition. This is possible because gangliosides and NgR [a GPI (glycosylphosphatidylinositol)-linked glycoprotein] are known to reside in cell surface signaling domains called lipid rafts and there are several examples of ganglioside-mediated modulation of receptor function (Miljan and Bremer, 2002). These and other issues of MAG signaling require additional investigations.

How do anti-ganglioside Abs induce inhibitory intracellular signaling through gangliosides that are restricted to the outer leaflet of the plasma membrane? Our current study does not examine this issue, but previous cell culture studies with multimeric Abs imply that ganglioside clustering is an important step in inhibition of nerve regeneration (Vyas et al., 2002). Our cur2b-treated animal
B
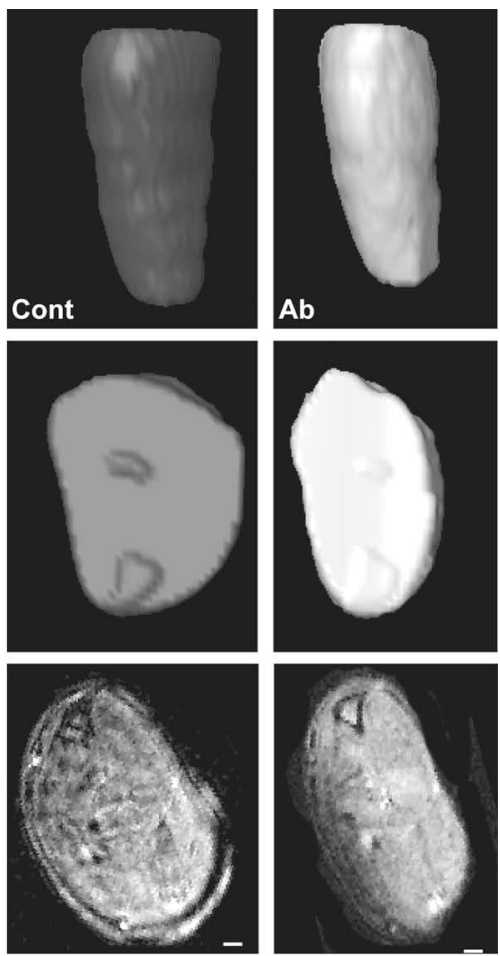

Figure 6. Anti-glycan Abs decrease target reinnervation of muscle. $A$, CMAP amplitudes recorded in hindpaws of mice at baseline and day 16 after nerve crush. GD1a/GT1b-2b-treated (Ab) animals have inexcitable nerves; sham Ab-treated (Cont) animals have delayed and dispersed CMAP amplitudes consistent with muscle reinnervation. $\boldsymbol{B}, 3 \mathrm{D}$ reconstructions of sham
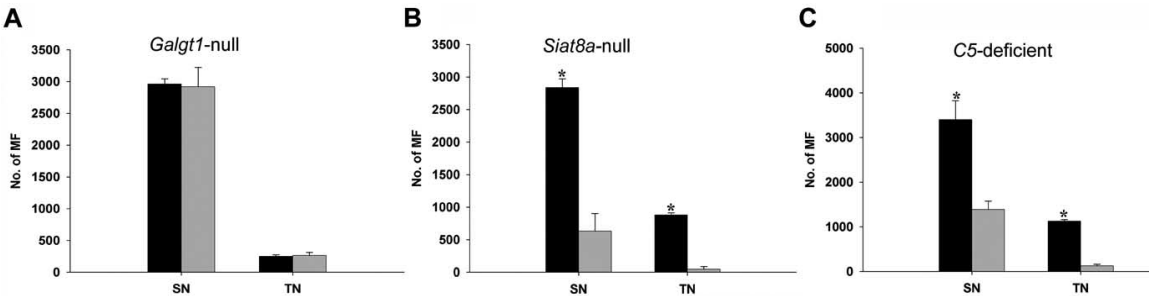

Figure 7. Effects of anti-glycan Abs on axon regeneration in mice with altered ganglioside or (5 expression. $\boldsymbol{A}$, Galgt1-null mice. Numbers of regenerating MF in GD1a/GT1b-2b-treated (gray bars; $n=3$ each) sciatic (2921 \pm 204$)$ and tibial $(262 \pm 51)$ nerves were similar to sham-Ab treated (black bars; $n=3$ each) sciatic (2963 \pm 79$)$ and tibial $(250 \pm 24)$ nerves. B, Siat8a-null mice. Numbers of regenerating MF in GD1a/GT1b-2b-treated (gray bars; $n=3$ each) sciatic (604 \pm 158 ) and tibial (43 \pm 23$)$ nerves. C, C5-deficient mice. Numbers of regenerating MF in GD1a/GT1b-2b-treated (gray bars; $n=4$ each) sciatic (1390 \pm 185$)$ and tibial (127 \pm 31$)$ nerves were significantly reduced compared with sham Ab-treated (black bars) sciatic $(3400 \pm 321)$ and tibial $(1127 \pm 31)$ nerves. ${ }^{*} p<0.05$. Error bars indicate SEM.

rent studies showed that inhibition of axon regeneration was induced by bivalent IgG anti-GD1a mAb. One potential explanation for these apparently divergent in vitro and in vivo findings is that Schwann cells are known to express Fc receptors (Vedeler et al., 2001) that can bind IgG Abs. This raises the possibility that in our animal model at least a fraction of GD1a/GT1b-2b Ab is bound to Schwann cells by Fc receptors and that regenerating axons in nerves encounter at least a fraction of $\mathrm{Ab}$ bound to Schwann cells in multimeric form that can cluster gangliosides. Another possibility is that the affinity of GD1a/GT1b-2b mAb is higher than those used in previous studies.

It has been proposed that ganglioside clustering triggers activation of small GTPase RhoA (Vinson et al., 2001), which partitions with gangliosides into lipid rafts (Simons and Toomre, 
2000). Initial evidence implies that ganglioside clustering recruits a transmembrane adaptor protein, p75 low-affinity NGF receptor, to lipid rafts for initiating the inhibitory signal transduction via RhoA (Fujitani et al., 2005). Whether other adaptor proteins are involved, or this inhibitory signaling can be induced independent of transmembrane adaptor protein (Hakomori, 2002), remains an open question. Our preliminary work indicates that GD1a/GT1b-2b Ab inhibits neurite outgrowth in primary neuronal cultures and activation of RhoA signaling is involved (K. A. Sheikh et al., unpublished observations). Additional studies are required to elucidate the signaling pathways involved in antiganglioside Ab-mediated inhibition of axon outgrowth.

Autoimmune anti-glycan Abs with specificity for gangliosides, including GD1a, are associated with both acute and chronic PNS and CNS autoimmune disorders (Acarin et al., 1996; Sadatipour et al., 1998; Mata et al., 1999; Willison and Yuki, 2002; Hughes and Cornblath, 2005). There is strong evidence for molecular mimicry as a mechanism for induction of these antiganglioside Abs in GBS. Clinical data from multiple sclerosis and GBS imply that anti-ganglioside Abs can also be induced secondary to the nervous system injury (Sadatipour et al., 1998; Press et al., 2001), and some experimental and clinical studies suggest that these Abs can be produced secondary to traumatic injury to the nervous system (Schwartz et al., 1982; Mizrachi et al., 1983; Hayes et al., 2002). Regardless of the mechanism of Ab induction, our data suggest that inhibition of axon regeneration may be part of the pathobiological spectrum of circulating autoimmune antiganglioside Abs. The studies in $\mathrm{C} 5$-deficient mice indicate that inhibition of axon regeneration in our model can occur independent of complement-dependent cytolytic injury. This is in contrast to our previous findings indicating that GD1a/GT1b-2b $\mathrm{mAb}$ induced mild injury to intact nerve fibers in a complementdependent manner (Sheikh et al., 2004).

In disease settings, the circulating Abs can access the transected axons in injured nervous system because of associated breakdown in blood-nerve or blood-brain barriers. The effects of these Abs on axon regeneration in CNS disorders would be extremely difficult to distinguish from those of intrinsic myelin and proteoglycan inhibitors, but the adverse effects of these circulating Abs on axon regeneration are more likely to be manifested in peripheral nerve disorders because of their relative capacity to regenerate after injury. Injured peripheral nerves do not always regenerate completely and restore normal connectivity and function, as exemplified by acute monophasic neuropathic disorders such as GBS in which up to $20 \%$ of patients are disabled, including 10\% who cannot walk unaided (Rees et al., 1998; Hughes and Cornblath, 2005).

Recovery after an attack of GBS is a complex multifactorial event and anti-glycan Abs with GD1a specificity may not always be associated with poor prognosis, because individual outcome is determined by several contributory factors, including extent and site of axonal injury, affinity/avidity of the Abs, isotype of the Ab [which affects the half-life of circulating Ab (Koga et al., 2003)], and age-related regenerative capacity of the host (Black and Lasek, 1979).

The sciatic nerve crush model used here is relevant to GBS, because (1) in both demyelinating and axonal forms of this disorder, the single most important predictor of prognosis and recovery is the extent of axonal injury (Brown and Feasby, 1984; Feasby et al., 1986; Hadden et al., 1998); and (2) circulating Abs have access to the injured nerves in GBS because of breakdown of the BNB as indicated by deposition of IgGs in both axonal and demyelinating variants of GBS (Hafer-Macko et al., 1996a,b).
Clinical evidence indicates that in patients with axonal injury sprouting/regeneration contributes toward recovery (Albers et al., 1985; Kuwabara et al., 2001). The mechanisms underlying failure of axon sprouting/regeneration in GBS cases with delayed/ poor recovery are not clear, but our current findings provide one explanation for delayed/poor recovery. Because breakdown of blood-tissue barrier(s) is almost always associated with morphological neural injury, a broader implication of our findings is that circulating factors, including autoantibodies, can access the injured nervous system and adversely affect neural repair.

\section{References}

Acarin N, Rio J, Fernandez AL, Tintore M, Duran I, Galan I, Montalban X (1996) Different antiganglioside antibody pattern between relapsingremitting and progressive multiple sclerosis. Acta Neurol Scand 93:99-103.

Albers JW, Donofrio PD, McGonagle TK (1985) Sequential electrodiagnostic abnormalities in acute inflammatory demyelinating polyradiculoneuropathy. Muscle Nerve 8:528 -539.

Black MM, Lasek RJ (1979) Slowing of the rate of axonal regeneration during growth and maturation. Exp Neurol 63:108.

Brown WF, Feasby TE (1984) Conduction block and denervation in Guillain-Barré polyneuropathy. Brain 107:219-239.

Carpo M, Pedotti R, Allaria S, Lolli F, Mata S, Cavaletti G, Protti A, Pomati S, Scarlato G, Nobile-Orazio E (1999) Clinical presentation and outcome of Guillain-Barré and related syndromes in relation to anti-ganglioside antibodies. J Neurol Sci 168:78-84.

Cinader B, Dubiski S, Wardlaw AC (1964) Distribution, inheritance, and properties of an antigen, MUB1, and its relation to hemolytic complement. J Exp Med 120:897-924.

Domeniconi M, Cao Z, Spencer T, Sivasankaran R, Wang K, Nikulina E, Kimura N, Cai H, Deng K, Gao Y, He Z, Filbin M (2002) Myelinassociated glycoprotein interacts with the Nogo66 receptor to inhibit neurite outgrowth. Neuron 35:283-290.

Feasby TE, Gilbert JJ, Brown WF, Bolton CF, Hahn AF, Koopman WF, Zochodne DW (1986) An acute axonal form of Guillain-Barré polyneuropathy. Brain 109:1115-1126.

Filbin MT (2003) Myelin-associated inhibitors of axonal regeneration in the adult mammalian CNS. Nat Rev Neurosci 4:703-713.

Fujitani M, Kawai H, Proia RL, Kashiwagi A, Yasuda H, Yamashita T (2005) Binding of soluble myelin-associated glycoprotein to specific gangliosides induces the association of p75NTR to lipid rafts and signal transduction. J Neurochem 94:15-21.

Gong Y, Tagawa Y, Lunn MP, Laroy W, Heffer-Lauc M, Li CY, Griffin JW, Schnaar RL, Sheikh KA (2002) Localization of major gangliosides in the PNS: implications for immune neuropathies. Brain 125:2491-2506.

Goodfellow JA, Bowes T, Sheikh K, Odaka M, Halstead SK, Humphreys PD, Wagner ER, Yuki N, Furukawa K, Furukawa K, Plomp JJ, Willison HJ (2005) Overexpression of GDla ganglioside sensitizes motor nerve terminals to anti-GDla antibody-mediated injury in a model of acute motor axonal neuropathy. J Neurosci 25:1620-1628.

Guzman-Harty M, Warner JK, Mancini ME, Pearl DK, Yates AJ (1990) Effect of crush lesion on ganglioside radiolabelling patterns in rat sciatic nerve. J Neuropathol Exp Neurol 49:225-236.

Hadden RD, Cornblath DR, Hughes RA, Zielasek J, Hartung HP, Toyka KV Swan AV (1998) Electrophysiological classification of Guillain-Barré syndrome: clinical associations and outcome. Plasma Exchange/Sandoglobulin Guillain-Barré Syndrome Trial Group. Ann Neurol 44:780-788.

Hafer-Macko C, Sheikh KA, Li CY, Ho TW, Cornblath DR, McKhann GM, Asbury AK, Griffin JW (1996a) Immune attack on the Schwann cell surface in acute inflammatory demyelinating polyneuropathy. Ann Neurol 39:625-635.

Hafer-Macko C, Hsieh S-T, Li CY, Ho TW, Sheikh K, Cornblath DR, McKhann GM, Asbury AK, Griffin JW (1996b) Acute motor axonal neuropathy: an antibody-mediated attack on axolemma. Ann Neurol 40:635-644.

Hakomori SI (2002) Inaugural article: the glycosynapse. Proc Natl Acad Sci USA 99:225-232.

Hayes KC, Hull TCL, Delaney GA, Potter PJ, Sequeira KAJ, Campbell K, Popovich PG (2002) Elevated serum titers of proinflammatory cyto- 
kines and CNS autoantibodies in patients with chronic spinal cord injury. J Neurotrauma 19:753-761.

Hughes RA, Cornblath DR (2005) Guillain-Barré syndrome. Lancet 366:1653-1666.

Kawai H, Allende ML, Wada R, Kono M, Sango K, Deng C, Miyakawa T, Crawley JN, Werth N, Bierfreund U, Sandhoff K, Proia RL (2001) Mice expressing only monosialoganglioside GM3 exhibit lethal audiogenic seizures. J Biol Chem 276:6885-6888.

Koga M, Yuki N, Hirata K, Morimatsu M, Mori M, Kuwabara S (2003) Anti-GM1 antibody IgG subclass: a clinical recovery predictor in Guillain-Barré syndrome. Neurology 60:1514-1518.

Kuwabara S, Ogawara K, Mizobuchi K, Mori M, Hattori T (2001) Mechanisms of early and late recovery in acute motor axonal neuropathy. Muscle Nerve 24:288-291.

Liu BP, Fournier A, GrandPre T, Strittmatter SM (2002) Myelin-associated glycoprotein as a functional ligand for the Nogo-66 receptor. Science 297:1190-1193

Liu Y, Wada R, Kawai H, Sango K, Deng C, Tai T, McDonald MP, Araujo K, Crawley JN, Bierfreund U, Sandhoff K, Suzuki K, Proia RL (1999) A genetic model of substrate deprivation therapy for a glycosphingolipid storage disorder. J Clin Invest 103:497-505.

Lunn MP, Johnson LA, Fromholt SE, Itonori S, Huang J, Vyas AA, Hildreth JE, Griffin JW, Schnaar RL, Sheikh KA (2000) High-affinity antiganglioside IgG antibodies raised in complex ganglioside knockout mice: reexamination of GD1a immunolocalization. J Neurochem 75:404-412.

Mata S, Lolli F, Soderstrom M, Pinto F, Link H (1999) Multiple sclerosis is associated with enhanced B cell responses to the ganglioside GD1a. Mult Scler 5:379-388.

Miljan EA, Bremer EG (2002) Regulation of growth factor receptors by gangliosides. Sci STKE 2002:RE15.

Mizrachi Y, Ohry A, Aviel A, Rozin R, Brooks ME, Schwartz M (1983) Systemic humoral factors participating in the course of spinal cord injury. Paraplegia 21:287-293.

Murinson BB, Hoffman PN, Banihashemi MR, Meyer RA, Griffin JW (2005) C-fiber (Remak) bundles contain both isolectin B4-binding and calcitonin gene-related peptide-positive axons. J Comp Neurol 484:392-402.

Mutoh T, Tokuda A, Miyadai T, Hamaguchi M, Fujiki N (1995) Ganglioside GM1 binds to the Trk protein and regulates receptor function. Proc Natl Acad Sci USA 92:5087-5091.

Pan B, Fromholt SE, Hess EJ, Crawford TO, Griffin JW, Sheikh KA, Schnaar RL (2005) Myelin-associated glycoprotein and complementary axonal ligands, gangliosides, mediate axon stability in the CNS and PNS: neuropathology and behavioral deficits in single- and double-null mice. Exp Neurol 195:208-217.

Press R, Mata S, Lolli F, Zhu J, Andersson T, Link H (2001) Temporal profile of anti-ganglioside antibodies and their relation to clinical parameters and treatment in Guillain-Barré syndrome. J Neurol Sci 190:41-47.

Ramon y Cajal S (1928) Degeneration and regeneration of the nervous system (May RM, translator). London: Oxford UP.

Rees JH, Thompson RD, Smeeton NC, Hughes RA (1998) Epidemiological study of Guillain-Barré syndrome in south east England. J Neurol Neurosurg Psychiatry 64:74-77.

Sadatipour BT, Greer JM, Pender MP (1998) Increased circulating antiganglioside antibodies in primary and secondary progressive multiple sclerosis. Ann Neurol 44:980-983.

Schafer M, Fruttiger M, Montag D, Schachner M, Martini R (1996) Disruption of the gene for the myelin-associated glycoprotein improves axonal regrowth along myelin in C57BL/Wlds mice. Neuron 16:1107-1113.
Schwartz M, Spirman N (1982) Sprouting from chicken embryo dorsal root ganglia induced by nerve growth factor is specifically inhibited by affinitypurified antiganglioside antibodies. Proc Natl Acad Sci USA 79:6080-6083.

Schwartz M, Sela BA, Esher N (1982) Antibodies to gangliosides and myelin autoantigens are produced in mice following sciatic nerve injury. J Neurochem 38:1192-1195.

Seitz RJ, Reiners K, Himmelmann F, Heininger K, Hartung H-P, Toyka KV (1989) The blood-nerve barrier in Wallerian degeneration: a sequential long-term study. Muscle Nerve 12:627-635.

Sheikh KA, Sun J, Lui Y, Kawai H, Crawford TO, Proia RL, Griffin JW, Schnaar RL (1999) Mice lacking complex gangliosides develop Wallerian degeneration and myelination defects. Proc Natl Acad Sci USA 96:7532-7537.

Sheikh KA, Zhang G, Gong Y, Schnaar RL, Griffin JW (2004) An antiganglioside antibody-secreting hybridoma induces neuropathy in mice. Ann Neurol 56:228-239.

Silver J, Miller JH (2004) Regeneration beyond the glial scar. Nat Rev Neurosci 5:146-156.

Simons K, Toomre D (2000) Lipid rafts and signal transduction. Nat Rev Mol Cell Biol 1:31-39.

Sparrow JR, Mcguinness C, Schwartz M, Grafstein B (1984) Antibodies to gangliosides inhibit goldfish optic nerve regeneration in vivo. J Neurosci Res 12:233-243.

Svennerholm L (1994) Designation and schematic structure of gangliosides and allied glycosphingolipids. Prog Brain Res 101:XI-XIV.

Tom VJ, Steinmetz MP, Miller JH, Doller CM, Silver J (2004) Studies on the development and behavior of the dystrophic growth cone, the hallmark of regeneration failure, in an in vitro model of the glial scar and after spinal cord injury. J Neurosci 24:6531-6539.

van Echten G, Sandhoff K (1993) Ganglioside metabolism. Enzymology, topology, and regulation. J Biol Chem 268:5341-5344.

Vedeler CA, Myhr KM, Nyland H (2001) Fc receptors for immunoglobulin $\mathrm{G}$-a role in the pathogenesis of Guillain-Barré syndrome and multiple sclerosis. J Neuroimmunol 118:187-193.

Vinson M, Strijbos PJ, Rowles A, Facci L, Moore SE, Simmons DL, Walsh FS (2001) Myelin-associated glycoprotein interacts with ganglioside GT1b. A mechanism for neurite outgrowth inhibition. J Biol Chem 276:20280-20285.

Vyas AA, Patel HV, Fromholt SE, Heffer-Lauc M, Vyas KA, Dang J, Schachner M, Schnaar RL (2002) Gangliosides are functional nerve cell ligands for myelin-associated glycoprotein (MAG), an inhibitor of nerve regeneration. Proc Natl Acad Sci USA 99:8412-8417.

Wang KC, Kim JA, Sivasankaran R, Segal R, He Z (2002) P75 interacts with the Nogo receptor as a co-receptor for Nogo, MAG and OMgp. Nature 420:74-78

Willison HJ, Yuki N (2002) Peripheral neuropathies and anti-glycolipid antibodies. Brain 125:2591-2625.

Yu RK, Saito M (1989) Structure and localization of gangliosides. In: Neurobiology of glycoconjugates (Margolis RU, Margolis RK, eds), pp 1-42. New York: Plenum.

Yuki N, Yamada M, Sato S, Ohama E, Kawase Y, Ikuta F, Miyatake T (1993) Association of IgG anti-GD1a antibody with severe Guillain-Barré syndrome. Muscle Nerve 16:642-647.

Zhang G, Lopez PH, Li CY, Mehta NR, Griffin JW, Schnaar RL, Sheikh KA (2004) Anti-ganglioside antibody-mediated neuronal cytotoxicity and its protection by intravenous immunoglobulin: implications for immune neuropathies. Brain 127:1085-1100. 